\title{
Going for growth: improvement in the infrastructural and management support for clinical academic research
}

\author{
Sally Fowler Davis \\ Sheffield Teaching Hospitals NHS Foundation Trust
}

\begin{abstract}
Our objective was to implement a directorate research strategy to improve and grow clinical academic capacity and capability and ensure that the organisational systems and processes enabled clinical staff and managers to increase grant capture, undertake clinically relevant research, including the adoption of NIHR portfolio sites and established a culture in which research was an accepted part of professional practice.
\end{abstract}

An initial evaluation of senior and middle manager attitudes and understanding of the research infrastructure and benefits of research identified that the directorate had a deeply segmented view of research and only a partial view of how research could benefit patients and improve their services. A significant number of staff claimed to be research active but this activity was not contributing to the service knowledge or being translated into grant capture, leading to income that could be used to invest in patient facing research. Few managers had appreciated the challenge of implementing the research strategy or the potential of enabling research active staff to generate clinical academic careers.

A quality improvement methodology was adopted, based on four equally important elements [1]; involving people (staff and patients) in research, developing people's research knowledge and skills, promoting an understanding of the complex systems and processes associated with research, and using an organisational research strategy with leadership to drive change. This improvement method suggests an equal and proportional range of activity to engage staff, amend and adapt processes and systems, carry out organisational change and "make it a habit". The improvement measures were selected by a number of managers who acted as "research champions" and shared these with all staff across the directorate; the focus was on delivering sustained improvements in performance targets agreed with the organisation. The interventions were introduced to assist managers in each professional group to champion research and undertake the organisational change that would be needed.

The two cycles of improvement over 14 months were used to achieve "academic status" within the organisation, to include clinical staff in the delivery of the research strategy and to create a clinical academic infrastructure that incorporated a well organised public and patient involvement (PPI) panel. Additional measures included the level of participation of clinical academic staff in grant capture and the level of income from research to sustain the growth in activity.

\section{Problem}

There is evidence that organisations where the research function is fully integrated into the organisational structure, tend to outperform other organisations that "pay less heed" to research outputs[2] and a plethora of policy related to clinical academic careers[3] recognised that many of the mechanisms to build research capacity; generate research proposals, capture grant income and deliver research studies were not fully developed. While a number of staff where aware of benefits of research on healthcare performance they were not able to deliver tangible research outputs.

A sustained focus on research impact is ongoing and further infrastructure is needed to engage AHPs in shaping research priorities.[4] There is a paucity of information nationally about nursing, midwifery, and AHP research capacity and capability with no clear indication of the number of nurses, midwives, and AHPs with research skills and qualifications. As the Finch Report made clear,[5] the number of nurses, midwives, and AHPs who have the qualifications and skills to lead research has remained low. No clear pathway has previously existed to combine clinical and academic careers. While all multi-professional staff should be competent evidence based practitioners, only some will be research active and fewer still will be research leaders.

\section{Background}

The directorate is a micro system within a teaching hospital with financial autonomy and discretion about how research is managed and developed. The vision of the clinical leadership was to achieve academic status that would be measured within the organisation and align to clinical academic career frameworks for nursing and allied health professions.[6] A researcher immersed in a clinical role is more likely to understand what research questions matter to the service and to the patient, to make those questions applicable to day to day clinical practice and to interpret research findings in the most practically useful way. A first cycle of improvement involved 
defining clinical academic as:

"Typically a parallel research and clinical activity investigating new ways of delivering better outcomes for patients with research grounded in the day to day clinical issues."

A first cycle of improvement involved engaging managers and identifying the baseline number of clinical academics or research capability in the directorate. We interviewed department leads to identify their commitment to research and to share the vision with the senior teams and make proposals for the learning/design activity. We discovered a number of issues that were seen as a barrier to further growth in research capacity. These included:

\section{- Managers' focus on operational activity across multiple sites and specialties}

- Managers' concern that research was a time consuming and individualised activity for the few

\section{- A poorly articulated link between clinical effectiveness and scholarly activity and research}

- An underdeveloped research infrastructure and capability in patient engagement, consultation, and partnership

- Limited workforce flexibility in relation to supporting fractional posts and backfill for research

- A perceived need to develop translational research in addition to the more traditional outputs.

The overall focus on individual, discretionary research activity tended to stretch individuals without delivering the outcomes in services or in terms of traditional impact of research. An initial intervention aimed to engage professional service managers to operationalise the directorate research strategy and reflect on the opportunity to increase research capacity and lead research as a group of research champions. They were also asked to contribute to the development of a set of baseline measures by which growth of clinical academic research could be measured, including staff engagement, research income, and publications.

\section{Baseline measurement}

The objective was to combine the tools and techniques of quality improvement with effective organisational and leadership development to achieve research capacity building. The quality improvement methodology[1] draws on a breadth of knowledge and research including technical engineering theories of systems, theories about human relationships and social interactions, and complexity theory.[7] Making organisational change demanded an understanding of complex adaptive systems $[8,9]$ and an appreciation of the complexity of the ways that structures, processes, and patterns work together as part of a system to generate different outcomes. Commitments to four equally important elements of improvement are a fundamental requirement; to identify drivers for change and also harness known mechanisms to achieve the improvement. Figure 1 identifies how the elements are highly intertwined and interconnected, and improvement to research activity and outcomes is regarded as a challenge to the system [10] and not to individual practitioners and clinicians

(See figure 1).

A project initiation document (PID) was written to identify the method and measures for a second cycle of improvement. This focused on identifying baseline data for research, gaining an inclusive understanding of the research strategy and re-designing research processes. Underpinning the research strategy was a commitment to changing patterns of thinking and behaviours associated with research. We wanted to promote clinical academic conversations, relationships, communication and learning, and decision-making along with recognition of the need to extend the engagement with patients as partners in the research process. This element of genuinely seeking patterns of change is important because the clinical academic role is unfamiliar to many and despite changes to structures and processes to support research, might become a barrier to change.[9]

The project initiation document identified the objective for the second cycle of change and these were agreed by managers and staff and to other key stakeholders.

\section{Objectives for second cycle of change}

1. To ensure that all staff in the directorate recognise the importance of research in relation to high quality patient care

2. To establish and re-enforce research collaborations to enable tendering and income generation

3. To identify $10 \%$ of staff engaged in clinical research (through tendering, delivering portfolio and no portfolio studies, and writing for publication)

4. To monitor a range of outputs and KPI's associated with academic directorate status

5. To establish continuous learning and knowledge exchange through networking and knowledge exchange for organisational benefit.

There were no baseline measures for research in the directorate and so the second cycle was designed to establish a set of measures that would be recognised within the organisation and become the key performance indicators for the achievement and impact of research activity. The measures were aligned to the NIHR conceptual framework [11] to provide a balanced score card and to enhance health research as a means of achieving clinical academic outputs and to demonstrate achievement of the strategic objectives associated with research. The objectives for the first year included key metrics associated with the engagement of $10 \%$ of clinical staff as academic participants and leaders and $80 \%$ of staff aware of the importance of research to patients and service outcomes. These indicators of staff engagement necessitated a further investigation into the clinical academic objectives within job roles and the indicators for success in capacity building and research outcomes were agreed to be: 
BMJ Quality Improvement Reports

- The level of patient recruitment to research studies

- The number of portfolio studies opened

- The number of grant applications

- The value of grant capture based income to the directorate

- The range of successful academic collaborations,

- The number of publications and conference presentations

Whilst these measures did not include specific translational benefits in terms of impact or benefits to patients and services, the focus on these metrics was deemed by the organisation to demonstrate a clinical academic environment and the academic status would indirectly benefit services and patient outcomes through research (see table).

See supplementary file: ds5881.pptx - "2 slides, the first the framework for improvement and the second are the baseline measures"

\section{Design}

The design of the improvement focussed on the staff and manager involvement and an intervention to help clinical academics navigate the development of a research proposal and gain managerial support for their research as core to their clinical practice. Managers were unaware of the local infrastructure to support research and therefore a commitment was made to develop the clinical academic infrastructure and to the invest in a 0.9 full time employee "clinical research officer" (band 8b) to navigate and codevelop research and a 0.5 full time employee directorate "patient and public involvement coordinator" to promote user/patient involvement in research. This investment was achieved in collaboration with the local collaborative research network (LCRN) and though a shared commitment with a partner University, with both organisations contributing to the cost of the new posts.

The following improvement activities were undertaken:

1. A process map was designed to inform all clinical academics about the sequence of tasks associated with developing a research proposal

2. Academic/University partnership and PPI structures were established through a series of formal meetings to harness collaboration. These were held four times during the year

3. Identification and mentoring of clinical academics who were ambitious to apply for NIHR funding for fellowships and grant proposals

4. Establish a Research Implementation Group for managers and research champions to change patterns associated with managing clinical academic activity.

The directorate established formal links with the clinical research office and engaged with existing systems and processes to make existing research activity more visible and allow access to training, governance, and development structures.

\section{Strategy}

The improvement methodology was adopted to guide the cycle of change and to collect data associated with research outcomes and achievement:

Involving people, staff, and patients: Three new groups were instituted, an academic board with professional leads associated with professional disciplines in the directorate including psychology, occupational therapy, speech, and language therapy, and physiotherapy. A research implementation group (RIG) of professional leads, research active staff, and newly established posts (public and patient involvement and clinical research officer) was constituted to deliver the research operational plan, with leadership devolved to a research lead and professional research "champions". A PPI panel was recruited and all participants offered development to respond to research proposals.

Developing people, staff, and patients: A commitment to an inclusive and cross professional research capacity building initiative was developed including events associated with the NIHR research design service and PPI funding, a collaboration with a University investing in wider participation from the local collaborative research network and writing for publication support. In addition, individual clinical staff were invited to identify clinically focused research questions, career aspirations, and involvement in portfolio studies with training in site management and patient recruitment and good clinical practice training (GCP). Relationships with the research workforce have been beneficial in so far as new processes and adaptations to accommodate new researchers have been developed; for example central collection of publication rates and citations.

Understanding complex systems and processes: The focus on workforce management including work planning for existing clinical staff in research and new opportunities was one priority and the other was the need to align new activity to the clinical research governance systems and processes within the organisation. A commitment to registering studies using a specified number was established for all new studies including potential bids, student studies and new portfolio sites. In addition, financial processes were refined to establish a cost code for research at directorate level and for new income for research posts, accrual income from the CRN and any research capacity funding. Furthermore a specific trawl of the NIHR portfolio of clinical studies was undertaken to identify possible site opening and principal investigator $(\mathrm{PI})$ opportunities for staff.

Organisational capacity and leadership: Sustaining the intention to develop research as a clear initiative that would contribute to service innovation and quality improvement was continually reiterated in the strategic aim and was re-articulated in artefacts and communications to staff and patients throughout the year. Research leadership was recognised in research champions who were enabled to develop new academic research collaborations through local contacts and the research design service and in partnership 
with the Yorkshire and Humberside Collaborations for Leadership in Applied Health Research and Care (CLAHRC)

All the mechanisms for driving and achieving improvement were shared across the directorate in professional service communications and via research champions, in association with the established systems of research governance and NIHR workforce guidelines using agenda for change criteria to establish new clinical academic posts.

\section{Results}

In March 2015, the directorate achieved academic status in recognition of the growth in research capacity and outputs and the development of a clinical academic infrastructure to support research and innovation. This clinical academic recognition now brings research capacity funding (RCF) which together with grant income and income from new portfolio sites and patient recruitment will make the research infrastructure sustainable. As a result the PPI coordinator post and clinical research officer post have been continued. In addition a fractional academic director role and the PPI panel membership can be maintained from the same budget line, releasing capacity for further clinical academic developments (see table).

Patient representatives and clinical staff have reported a real shift in the perception of research as a valued and patient/service development activity with managers supporting initiative and collaborations with academic partners. Over 14 months eight clinical staff have applied for the NIHR funded masters in clinical research and six have been accepted. In addition five clinical staff have applied for NIHR clinical academic fellowships with two being successful. These individual achievements represent the importance of developing an organisational approach to improving the clinical academic research infrastructure to promote careers and sustain the research strategy.

Most notably, further investment and partnership with universities has shown real benefits in terms of additional and sustained capacity building and this has resulted in partnership proposals for European funding applications and NIHR themed calls.

See supplementary file: ds5882.pptx - "Table 2 Results of Intervention"

\section{Lessons and limitations}

The introduction of quality improvement as an approach to capacity building is new and was 'untested' at the start of the process. The use of organisational metrics was introduced part way through the process but aligned well with the key performance indicators that had been established within the directorate. Clinical staff engagement beyond the initial champions was slow to develop as was the academic collaboration with senior professorial leads who were time-limited and initially unconvinced that the ambition could be achieved in a short time scale. Their view of early career researchers, mirrored by the novice clinical academic workforce themselves, was that the ambition had been well conceived but was not manageable within a 12 to 14 month period. This skepticism has been somewhat challenged by the achievement of academic status and the level of achievement in clinical academic activity across professions in the directorate.

Some critical problems remain; that the relatively early career clinical academics require support and guidance in the complex and ever-changing range of opportunities and barriers to developing clinical academic activity. Continuing investment in the PPI and research officer roles has been necessary to achieve the operational plan. In addition, the clinical pressure on staff and managers remains and increases, continuing the tension between investments in research as a quality improvement initiative in medium term with the highly present short term challenge of delivering care and services. Proposed interventions (such as the learning and development events) have not recruited well and it has been difficult to offer specific training in groups.

Professional management have achieved a far greater knowledge and understanding of the opportunities for research to compliment the clinical workforce and workforce flexibility, capacity, and contribution of partners in academic practices has been a tangible benefit and fully costed. Compliance with the research strategy and operational improvements were based on a principle of inclusivity and engagement and this has been thoroughly carried through with an individual response to all clinical researchers and a focus on the voice of the patient in research and a compliance with the INVOLVE principles.[12] A significant limitation of the project as a whole and the metrics adopted by the organisation to measure research outputs is the lack of translational measures that might demonstrate innovation and impact in patient care.

\section{Conclusion}

Research remains an important and current policy initiative within the NHS and there is a growing evidence to suggest that research and a research active workforce is associated with better patient outcomes and experiences. This improvement in research capacity in a single hospital directorate demonstrates the importance of addressing professional management concerns about the need to plan and sustain research against a back drop of clinical performance and effectiveness. Achievements were based on individual commitment and an improvement to the research environment based on collaboration and meeting infrastructure to support an operational plan. Enabling existing and new clinical staff to become academically active has been developed using systems and process development that managed the risk of undertaking further work in an uncosted and unsustainable way. In addition a greater shared understanding of the research landscape, including the NIHR support through the research design service and partnership with local universities has been forthcoming to enable workforce capacity.

Clinical management involvement, especially professional and service managers is key to achieving the improvement and the new research capacity. Managers were able to advise on workforce developments and achieve the operational changes. Equally, they 
require a clear plan for the funding and investment in research and demand a clear plan to cost and sustain any investment. In addition, investment in clinical academic career developments including new posts, NIHR applications and development programmes are important and this quality improvement has sought to achieve a wide systems and process alignment with the wider research landscape in order to achieve. It is now an imperative to maintain the necessary support for participation in clinical research - ethical logistical and financial [13] so that the impact and benefits are not limited to participants but to the knowledge of the organisation as a whole, to bring the benefit to patient outcomes and to capture system wide benefits overall.

\section{References}

1. Penny J. 'Discipline of Improvement in Health and Social Care' in, NHS Institute for Innovation and Improvement 2003 'Improvement Leaders' Guide: Improvement Knowledge and skills', NHS Institute for Innovation and Improvement 2005

2. Hanney S, Boaz A, Jones T, Soper B. Engagement in research: an innovative three stage review of the benefits for health care performance NIHR HSRD 010802014

3. NHS England: Clinical Academic Careers Framework: A framework for optimising clinical academic careers across healthcare professions 2014 at http://hee.nhs.uk/wp-content /blogs.dir/321/files/2014/07/2014-12-CACFramework-21-1-15.pdf (accessed 12.4.2015).

4. Pickstone C, Nancarrow S, Cooke J, Vernon W, Mountain G, Boyce RA, Campbell J. Building research capacity in the allied health professions. Evidence and Policy: A Journal of Research, Debate, and Practice 2008;4(-1):53-68.

5. UK Clinical Research Collaboration: Developing the Best Research Professionals. Qualified graduate nurses: recommendations for preparing and supporting clinical academic nurses of the future. Report of the Subcommittee for Nurses in Clinical Research (Workforce). UKCRC[Dame Janet Finch] 2007.

6. Department of Health: Developing the Role of the Clinical Academic Researcher in the Nursing, Midwifery and Allied Health Professions, London: 2012 at http://www.dh.gov.uk/prod consum dh/groups/dh digitalass ets/@dh/@en/documents/digitalasset/dh_133094.pdf

7. NHS Institute for Improvement and Innovation:Improvement leaders Guides -Personal and Organisational Development 2005

http://www.institute.nhs.uk/building capability/building improvemen t_capability/improvement_leaders'_guides\%3A_pers.html accessed 5th June 2015

1. Gell-Mann M. The quark and the jaguar-adventures in the simple and the complex, Abacus 1994.

2. Plsek $P$, Greenhalgh $T$. The challenge of complexity in healthcare. BMJ 2001;323:625-8.

3. Maher L, Penny J. Service Improvement, in Peck, E 'Organisational Development in Healthcare: approaches, innovations and achievement', 2005Oxford: Radcliffe publishing.

4. El Turabi A, Hallsworth M, Ling T, Grant J. A novel performance monitoring framework for health research systems: experiences of the National Institute for Health Research in England. Health Res Policy Systems 2011;9(13):505-13.

5. NIHR, INVOLVE at http://www.invo.org.uk/posttyperesource/where-and-how-toinvolve-in-the-research-cycle/ access 12.7.15.

6. Kzyzanowska MK, Kaplan R, Sullivan S. How may clinical research improve healthcare outcomes? Anns Oncol $2011 ; 22(7): 10-5$

\section{Declaration of interests}

None to declare.

\section{Acknowledgements}

Rev Dr Mark Cobb, clinical director therapeutic and palliative services directorate and Dr Sue Pownall, academic director.

\section{Ethical approval}

According to the policy activities that constitute research at Sheffield Teaching Hospitals Trust this work met criteria for operational improvement activities exempt from ethics review. We used the following criteria for determining if improvement activities require ethics review. Policy criterion: The work is primarily intended to improve local organisational processes, not provide generalisable knowledge in a field of inquiry. 